\title{
Why is $G$ the Least Precisely Known Physical Constant?
}

\author{
C. C. Speake \\ Bureau International des Poids et Mesures, Pavillon de Breteuil, F-92312, Sèvres Cedex, France
}

G. T. Gillies

University of Virginia, Department of Nuclear Engineering and Engineering Physics, Charlottesville, Virginia 22901, USA

Z. Naturforsch. 42 a, 663-669 (1987); received January 23, 1987

\begin{abstract}
CODATA has recently published its readjustment of the fundamental physical constants and assigns a relative precision of $128 \times 10^{-6}$ to $G$, the Newtonian constant of gravitation. Given that most of the other constants in physics have relative precisions of $\sim 10^{-6}$ or better, we examine the reasons why the value for $G$ remains so imprecise: The role of $G$ in physics in general is considered and the most recent experimental determinations are examined. Constraints are given for perturbing effects in $G$ measurements and a key result is that horizontal ground movements must be taken more carefully into account in future more precise terrestrial experiments.
\end{abstract}

\section{The Role of $G$ in Physics}

In physical science there are very few subject areas which demand a precise value for $G$, the Newtonian constant of gravitation. In celestial mechanics, for instance, it is only the products of planetary masses and $G$ which are of importance in determining orbital parameters. Another example arises in geophysics, where the most important "constant" is $g$, the acceleration of gravity at a point on the earth's surface, a quantity which includes, again, $G$, the mass of a planet (earth), and the radius of the earth. Most scientists and engineers simply have no need of $G$ in their work.

There are, however, some fields of study which require its use. In general relativity $G$ is a factor in the constant which relates stress-energy of space time to its curvature. Then, in astrophysics, the luminosity of stars depends on the seventh power of $G$. Moreover, in cosmology, our present interpretations of Mach's principle state that the magnitude of local inertial forces is governed by the product of $G$ and a factor determined by the large-scale mass distribution of the universe. A numerical value for $G$ can, in principle, be derived using Mach's principle.

Values of $G$ have also been derived from other theoretical standpoints, as well. Although there is,

Reprint requests to Dr. C. C. Speake, Bureau International des Poids et Mesures, Pavillon de Breteuil, F-92312 Sèvres Cedex, Frankreich as yet, no consensus on even the fundamental principles used as a starting point for such calculations, several workers $[1,2,3]$ have produced useful theoretical models that may lead to $G$, and some have actually derived numerical results $[4,5]$ which are in agreement with the currently accepted measurements [6]. In much the same way that quantum electrodynamics has driven refinements in the measurements of the fine structure constant, a well-accepted theoretical prediction of $G$ would provide a stirring stimulus for higher-precision gravity experiments. Furthermore, such a prediction would relate $G$ to the atomic constants, thereby increasing its importance to metrologists as well. An alternative viewpoint is that $G$ should be given a fixed value: a combination of $G, c$, and Planck's constant, $h$, could be used to define the unit of mass, the Planck mass, in a way similar to the 1983 definition of the metre in terms of a fixed value for the speed of light [7]. Indeed, it seems likely, according to superstring theories [8], that the masses of the elementary particles can be computed from the Planck mass. It is interesting to compare the relative rates of increase in the precision of measurements of $G$ and $c$ as shown in Figure 1. Whilst there was no stimulus from theoretical physics to increase the precision of the speed of light, the same is not necessarily the case for the gravitational constant. The relentless increase in the precision of the speed of light was fuelled by advances in technologies such as radio-frequency techniques and lasers. In contrast, 


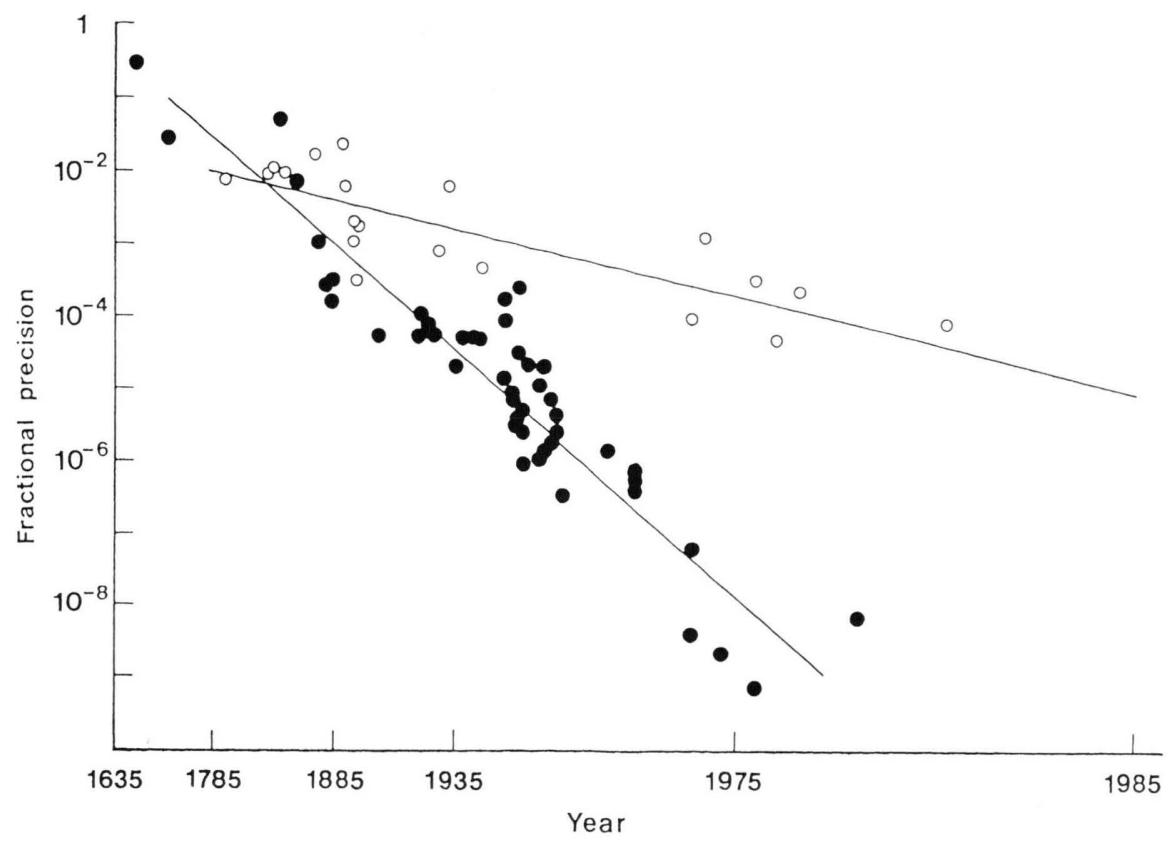

Fig. 1. The increase in fractional precision of the Newtonian constant of gravitation $G$ and the speed of light, $c$ (finally fixed by definition in 1983 [7]). The $\log -\log$ plot clearly shows that the rate of reduction of the uncertainties of these fundamental constants is not linear in time; the $c$ curve can be parametrised as $\Delta c / c \alpha t^{-4.9}$ and $\Delta G / G \propto t^{-1.3}$ where $t$ is time in years. To keep pace with the increase in precision predicted by the $G$ curve, we must achieve an improvement of an order of magnitude within the next six years! (The data used for this plot can be found in [45].)

there have been no such breakthroughs in the measurement of gravitational forces since the time of Cavendish.

We feel that a strong motivation for continued development of the techniques of experimental gravitation comes from the present drive to unify the four forces of nature [9]. One of the earliest attempts to incorporate gravity into a unified theory was made by Fujii $[10,11]$, who proposed breakdowns in the Newtonian description of gravity and related them to other couplings via a scale-invariant theory. This led to a variety of experimental verifications of the inverse square law. On geophysical scales, however, the work of Holding et al. [12] would suggest that this question is still open, and some see their results as possible evidence for an additional short range force in nature [13]. The theoretical development continued with contributions from Feinberg and Sucher [14]. Later, Gibbons and Whiting [15] established the presently accepted parametrization of the non-Newtonian component of such a force.
In parallel with the theoretical and experimental interest in $G(r)$ there evolved an interest in the possibility of a time dependence of $G$. The original suggestion of Dirac [16] was restudied by several workers during the 1970's toward the goal of incorporating his large-numbers hypothesis as a gauge condition in a scale-covariant theory of gravity. Moreover, the possibility of radar ranging to the inner planets presented the hope of experimental investigation [17]. Unfortunately, the results of these experiments are not unambiguous [18], and a definitive laboratory experiment is not yet foreseen, although there exists at least one serious candidate [19]. A recent study [20] has given the exciting result that a certain class of superstring theories predict a value of the fractional rate of change of $G$ as $-1 \times 10^{-11 \pm 1}$ per year. Unambiguous, non-astronomical measurements of this quantity are required to distinguish between these theories.

Nevertheless, to the present day, it appears that gravitation stands apart from the quantized field theories, and in such a decoupled theoretical picture 
there is no doubt that $G$ is, in fact, a true constant of nature. An incorporation of gravitation into the rest of physics, with the resultant unification of the forces, is the ultimate goal of theoretical physics, and developments such as the superstring theories and the new inflationary universe scenario give real hope of reaching this goal.

\section{The Experimental Determination of $G$}

In its recent reassessment of the values of the fundamental constants [21], CODATA gives a value for $G$ as

$$
G=(6.67259 \pm 0.00085) \times 10^{-11} \mathrm{~m}^{3} \mathrm{~kg}^{-1} \mathrm{~s}^{-2} .
$$

The relative uncertainty in this value is $128 \times 10^{-6}$, and this result is based on an analysis of the three most recent measurements [6], each of which claims an uncertainty of about $10^{-4}$, but all of which exclude each other from the limits of error quoted by each of the authors. All three of these works involved the use of torsion pendulums: Sagitov et al. [22] and Luther and Towler [23] used the time of swing method largely developed by Heyl and Chrzanowski [24] whereas Pontikis [25] employed a resonance method similar to that first used by Zahradnicek [26].

We have attempted to summarize in Table 1 the possible perturbations encountered in an experiment to determine $G$ to a relative precision of $10^{-6}$. We have taken the attracting mass to be $10 \mathrm{~kg}(M)$, the test mass $(\mathrm{m})$ to be $10 \mathrm{~g}$ and their centre of mass separation $(r)$ as $10 \mathrm{~cm}$. These parameters are representative of the most recent torsion balance measurements.

The first four items in the table are straightforwardly calculated and the constraints listed in the final column can be satisfied in practice without too much difficulty. However, the problem of horizontal ground vibrations is of a more subtle nature and much more difficult to resolve: A horizontal acceleration $\left(\ddot{y}_{0}\right)$ will cause a simple pendulum oscillation of the torsion balance. The balance arm will then try to align itself perpendicularly to the direction of the ground displacement in order to minimise its moment of inertia (and kinetic energy) about the rotation axis. We have calculated the magnitude of this effect by assuming a simplified
Table 1. The constraints on perturbation effects in a determination of $G$ to a relative precision of $10^{-6}$. See the text for details of the experimental arrangement considered.

\begin{tabular}{|c|c|c|}
\hline Perturbing effect & Comments & Limits for $10^{-6} G$ \\
\hline $\begin{array}{l}\text { Gravity gradient } \\
\text { due to a person } \\
\text { of mass } 100 \mathrm{~kg}\end{array}$ & $\begin{array}{l}\text { torsion balance } \\
\text { armlength } 20 \mathrm{~cm}\end{array}$ & $\begin{array}{l}\text { closest distance of } \\
\text { approach } \lambda>25 \mathrm{~m}\end{array}$ \\
\hline $\begin{array}{l}\text { Electrostatic } \\
\text { forces }\end{array}$ & $\begin{array}{l}\text { grounded con- } \\
\text { ducting vacuum } \\
\text { chamber }\end{array}$ & $\begin{array}{l}\text { voltage difference } \\
\text { between test masses } \\
\text { and chamber } \\
\Delta V \leqq 5 \mathrm{mV}\end{array}$ \\
\hline $\begin{array}{l}\text { Thermomolecular } \\
\text { flow }\end{array}$ & $\begin{array}{l}\text { vacuum pressure } \\
10^{-5} \mathrm{~mm} \mathrm{Hg} \\
\left(1.3 \times 10^{-8} \mathrm{~Pa}\right)\end{array}$ & $\begin{array}{l}\text { temperature differ- } \\
\text { ence across chamber } \\
\Delta T \leqq 0.1 \mathrm{~K}\end{array}$ \\
\hline $\begin{array}{l}\text { Magnetostatic } \\
\text { forces }\end{array}$ & $\begin{array}{l}\text { no magnetic } \\
\text { shielding, pure } \\
\text { materials }\end{array}$ & $\begin{array}{l}\text { product of volume } \\
\text { susceptibilities of } \\
\text { masses } \chi_{1} \chi_{2} \leqq 10^{-7}\end{array}$ \\
\hline $\begin{array}{l}\text { Horizontal } \\
\text { ground } \\
\text { vibrations }\end{array}$ & $\begin{array}{l}\text { critical damped } \\
\text { simple pendulum } \\
\text { mode, white ac- } \\
\text { celeration spec- } \\
\text { trum (see appendix) }\end{array}$ & $\begin{array}{l}\text { spectral density of } \\
\text { horizontal accelera- } \\
\text { tion } \\
\ddot{y} \leqq 10^{-7} \mathrm{~m} \mathrm{~s}^{-2} / \mathrm{Hz}^{1 / 2}\end{array}$ \\
\hline $\begin{array}{l}\text { Thermo-elastic } \\
\text { coefficient } \\
\text { of fibre }\end{array}$ & $\begin{array}{l}\text { time of swing } \\
\text { method with } \\
35 \mu \mathrm{m} \text { diameter } \\
\text { tungsten fibre of } \\
\text { thermo-elastic co- } \\
\text { efficient } \beta=7 \times 10 \\
\text { (for quartz } \\
\beta=-1.1 \times 10^{-4} \text { ) }\end{array}$ & $\begin{array}{l}\text { variations in ab- } \\
\text { solute temperature } \\
\Delta T_{\text {abs }} \leqq 0.01 \mathrm{~K}\end{array}$ \\
\hline
\end{tabular}

model of a torsion balance as described in the appendix, and we note that a complete treatment of the dynamics of the torsion balance can be found elsewhere [27].

Assuming a white horizontal vibration spectrum (which is realistic for frequencies higher than $\sim 1 \mathrm{~Hz})$ of magnitude $\ddot{y}_{0}(v)$ we find the rms value of the applied noise torque to be

$$
\left\langle\tau^{2}\right\rangle^{1 / 2} \simeq \ddot{y}_{0}(v)^{2} \frac{m d^{3 / 2}}{4 g^{1 / 2}} \frac{Q \sin 2 \psi}{\left(d^{2}+R^{2} \sin ^{2} \psi\right)^{1 / 2}},
$$

where $Q$ is the simple pendulum mode quality factor, $d$ is the length of the torsion fibre and $\pi / 2-\psi$ is the angle between the balance arm and the direction of the ground displacement (see Figure A 1). The maximum noise level which can be tolerated given in Table 1 has been calculated assuming that the simple pendulum mode is critically damped as was approximately the case in Luther and Towler's experiment. However, even under these circum- 
stances, the spectral density limit is close to the minimum that one can expect at a quiet location on our planet [28]. This result clearly implies that damping for the simple pendulum mode and vibration isolation are required in future determinations of $G$ with otherwise uncompensated torsion balances. Equally, as typical values of ground vibration level and the $Q$ of the undamped simple pendulum mode are $5 \times 10^{-5} \mathrm{~m} \mathrm{~s}^{-2} / \mathrm{Hz}^{1 / 2}$ and $10^{4}$, respectively, it is quite possible that this perturbation has limited the precision of previous determinations.

The coupling can be reduced by increasing the symmetry of the suspended system as has been done on the tests of the weak equivalence principle by Roll et al. [29] and Braginsky et al. [30]. Unfortunately, increasing the number of balance arms also reduces the gravity signal in a $G$ measurement as well as the sensitivity of the balance. Perhaps, even so, this approach should be investigated in more detail. Sagitov et al. noted that a fractional change of period of up to $1.5 \times 10^{-5}$ could occur between night and day-time measurements, and this variation, as they point out, was due to the changing amplitude of ground vibrations. The fluctuations in oscillation period gave rise to a fractional uncertainty in Sagitov et al.'s value of $G$ of $1.2 \times 10^{-4}$. It is interesting to note that in Pontikis' resonance method the statistical uncertainty associated with each individual measurement is only of the order of $10^{-5}$. However his set of $G$ values shows a clear time dependence of about $10^{-5}$ per day.

The time of swing method relies on the reproducibility of the torsion constant of the fibre, and it is this feature of the $G$ determinations which has proved the most troublesome in the past. Sagitov et al. observed variations in the period of oscillation of their tungsten fibre torsion balance due to changes in shear modulus as a function of temperature (see Table 1) and, taking their value for the thermo-elastic coefficient, a $35 \mu \mathrm{m}$ diameter fibre and our model experimental parameters, it can be shown that the temperature of the fibre must be held constant to $0.01 \mathrm{~K}$ to achieve a $10^{-6}$ determination of $G$. This would not be easy but is possible in principle.

The natural period of oscillation of the quartz fibre used by Luther and Towler drifted by a fractional amount of $3 \times 10^{-6}$ per day, and they attempted to eliminate this unexplained effect by removal of a trend from the data. The statistical variations of the residuals gave a relative uncertainty of $4 \times 10^{-5}$ to the value of $G$ which was the largest single contribution to the error budget. These problems with the stability of the fibre are most likely due to anelasticity and could be eliminated with feedback techniques of the type developed by de Boer et al. [31].

Another difficulty arises in the need for absolute distance measurements which requires that the position of the centres of mass of the bodies be well known: however density inhomogeneities make this uncertain. According to the error budgets of the recent experiments such metrological problems would limit the relative precision to about $4 \times 10^{-5}$.

New measurement strategies are being studied which may obviate some of the problems. For instance, various alternatives to the torsion-fibre suspension have been investigated. These include magnetic suspensions [32, 33] and fluid suspensions [31, 34]. Other forms of classical mechanical detector such as the compound pendulum [35] and the beam balance [36] are being revived and show promise of becoming serious alternatives to the torsion balance [37]. All these detectors aim at increasing the gravitational torque signal by increasing the mass of the suspended test bodies over that possible with torsion balances and use servo systems to improve the stability of the suspension. Feedback can also be used to isolate mechanical detectors from microseismic noise, a technique which is also being developed for interferometric gravity wave detectors [38]. Another key feature of this class of alternative detector is that they are less susceptible to perturbations due to ground vibrations.

Advances in the manufacture and measurement of the homogeneity of amorphous materials of the sort used in the Relativity Gyro Experiment [39] will reduce the uncertainties in the metrology of the attracting masses. Similarly, the use of monocrystalline silicon in a Cavendish experiment has already been proposed [40]. The difficulties associated with ground vibrations would be greatly reduced if an appropriate experiment could be performed in space. On the other hand, new design challenges would arise and the problems associated with them have been discussed by various authors $[41,42,43$, 44].

The route toward a more precise value of $G$ lies in long-term experimental programmes which address the problems associated with the terrestrial 
experiments and also investigate the difficulties of measurements in space. The experience and knowledge gained in searches for forces weaker than gravity of the type proposed by Fischbach et al. [13] may also contribute usefully to the techniques required in experimental gravitation.

We would like to thank W. Towler, T. J. Quinn, and R. C. Ritter for useful discussions. One of us (CCS) thanks the Centre for Advanced Studies of the University of Virginia for financial assistance in Support of this work.

\section{Appendix}

The torque on a torsion balance due to horizontal ground movements

For this calculation we imagine that the torsion balance comprises a light, rigid inverted " $T$ " attached to the ground by an ideal universal flexible joint at 0 (see Fig. A 1). The $y$ axis is defined by the direction of the horizontal ground displacement, $y_{0}$, which can of course lie at any arbitrary angle $(\pi / 2-\psi)$ to the suspended dumb-bell.

First we calculate the coordinates of the dumbbell after the arbitrary displacement shown in Fig. A2, and then go on to calculate the Lagrangian of the system. The initial positions of the two test masses are given by the vectors

$$
\pm R \cos \psi \boldsymbol{i} \pm R \sin \psi \boldsymbol{j}-\mathrm{d} \boldsymbol{k} .
$$

These vectors give the positions of the masses relative to the point $\left(0, y_{0}, 0\right)$ before the rotation in the $y-z$ plane of the pendulum "fibre". A rotation about the $x$ axis can be described mathematically by the transformation matrix,

$$
\mathbf{M}=\left(\begin{array}{ccc}
1 & 0 & 0 \\
0 & \cos \theta & -\sin \theta \\
0 & \sin \theta & \cos \theta
\end{array}\right),
$$

Thus the coordinates of the masses of the rotated pendulum relative to $\left(0, y_{0}, 0\right)$ become

$$
\left(\begin{array}{l}
x^{\prime} \\
y^{\prime} \\
z^{\prime}
\end{array}\right)=\mathbf{M}\left(\begin{array}{l} 
\pm R \cos \psi \\
\pm R \sin \psi \\
-d
\end{array}\right)
$$

or

$$
\left(x^{\prime}, y^{\prime}, z^{\prime}\right)=( \pm R \cos \psi, \pm R \sin \psi \cos \theta+d \sin \theta,
$$$$
\pm R \sin \psi \sin \theta-d \cos \theta \text { ). }
$$

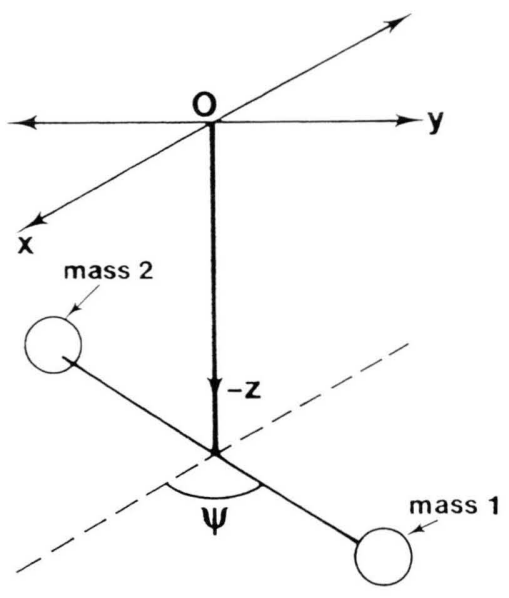

Fig. A1. Schematic of the model dumb-bell torsion balance and the coordinate axes. The "fibre" has a length $d$ and the total length of the balance arm is $2 R$.

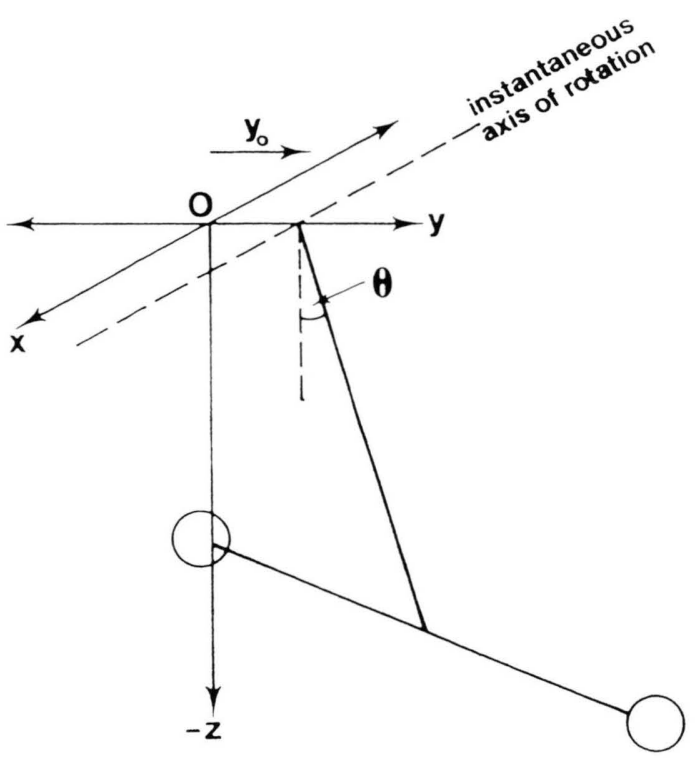

Fig. A2. A horizontal ground movement, $y_{0}$, gives rise to a simple pendulum oscillation of amplitude $\theta$ about the instantaneous axis of rotation.

So the coordinates of the two masses in the original coordinate system (relative to 0 ) are

$$
\begin{aligned}
(x, y, z)= & \left( \pm R \cos \psi, y_{0} \pm R \sin \psi \cos \theta+d \sin \theta,\right. \\
& \pm R \sin \psi \sin \theta-d \cos \theta) .
\end{aligned}
$$

We can now calculate the potential, $V$, and kinetic, $T$, energies of the dumb-bell masses. For 
mass 1 we have

$$
\begin{aligned}
T_{1}=\frac{m}{2}\left\{\dot{x}^{2}+\dot{y}^{2}+\dot{z}^{2}\right\} \\
=\frac{m}{2}\left\{\dot{y}_{0}^{2}+\dot{\psi}^{2} R^{2}+\dot{\theta}^{2}\left(d^{2}+R^{2} \sin ^{2} \psi\right)\right. \\
\quad+\dot{y}_{0} \dot{\theta}(2 d \cos \theta-2 R \sin \psi \sin \theta) \\
\left.\quad+\dot{y}_{0} \dot{\psi}(2 R \cos \psi \cos \theta)+\dot{\psi} \dot{\theta}(2 R \mathrm{~d} \cos \psi)\right\}
\end{aligned}
$$

and for mass 2 ,

$$
\begin{aligned}
T_{2}=\frac{m}{2}\{ & \dot{y}_{0}^{2}+\dot{\psi}^{2} R^{2}+\dot{\theta}^{2}\left(d^{2}+R^{2} \sin ^{2} \psi\right) \\
& +\dot{y}_{0} \dot{\theta}(2 d \cos \theta+2 R \sin \psi \sin \theta) \quad(\mathrm{A} 7 \\
& \left.-\dot{y}_{0} \dot{\psi}(2 R \cos \psi \cos \theta)-\dot{\psi} \dot{\theta}(2 R d \cos \psi)\right\},
\end{aligned}
$$

thus giving a total kinetic energy

$$
\begin{aligned}
T=m & \left\{\dot{y}_{0}^{2}+\dot{\psi}^{2} R^{2}+\dot{\theta}^{2}\left(d^{2}+R^{2} \sin ^{2} \psi\right)\right. \\
& \left.+2 \dot{y}_{0} \dot{\theta} d \cos \theta\right\} .
\end{aligned}
$$

The potential energy is simply

$$
\begin{aligned}
V & =m g\left\{z_{1}+z_{2}\right\} \\
& =-2 m g d \cos \theta,
\end{aligned}
$$

and the Lagrangian of the system is

$$
L=T-V \text {. }
$$

We can now calculate the equations of motion in $\theta$ using Lagrange's equation

$$
\frac{\mathrm{d}}{\mathrm{d} t}\left(\frac{\partial L}{\partial \dot{\theta}}\right)-\frac{\partial L}{\partial \theta}=0
$$

We find

$$
\begin{aligned}
\frac{\mathrm{d}}{\mathrm{d} t}\left(\frac{\partial T}{\partial \dot{\theta}}\right)=2 m\{ & \ddot{\theta}\left({ }^{2}+R^{2} \sin ^{2} \psi\right) \\
& +2 \dot{\theta} R^{2} \sin \psi \cos \psi \dot{\psi} \dot{\theta} \\
& \left.+\ddot{y}_{0} d \cos \theta-\dot{y} \dot{\theta} d \sin \theta\right\}
\end{aligned}
$$

and that

$$
\frac{\partial(-V)}{\partial \theta}=-2 m g d \sin \theta
$$

The equation of motion in $\theta$ then becomes

$\left(d^{2}+R^{2} \sin ^{2} \psi\right) \ddot{\theta}+R^{2} \sin 2 \psi \dot{\psi} \dot{\theta}^{2}$

$+\ddot{y}_{0} d \cos \theta-\dot{y} \dot{\theta} d \sin \theta+g d \sin \theta=0$ or ignoring third order and higher terms, we obtain

$$
\left(d^{2}+R^{2} \sin ^{2} \psi\right) \ddot{\theta}+g d \theta=-\ddot{y}_{0} d .
$$

For the $\psi$ equations of motion we find

$$
\frac{\mathrm{d}}{\mathrm{d} t}\left(\frac{\partial T}{\partial \dot{\psi}}\right)=2 m R^{2} \ddot{\psi},
$$

but

$$
\partial L / \partial \psi=2 m R^{2} \sin \psi \cos \psi \dot{\theta}^{2}
$$

and then obtain

$$
\ddot{\psi}-\frac{1}{2} \sin 2 \psi \dot{\theta}^{2}=0 \text {. }
$$

We notice that the simple pendulum oscillation can apply torques to the torsion mode of the pendulum. The basic mechanism is that of the reduction of the kinetic energy by the lining up of the dumb-bell with the axis of rotation. The simple pendulum oscillation can apply a fixed torque for $\psi=\pi / 4$ or apply an additional torque per unit deflection to the balance for $\psi \simeq 0$ or $\pi / 2$. We shall calculate the effect of horizontal ground vibrations on both the time of swing method and the static deflection method.

First we must find an expression for $\dot{\theta}^{2}$ in terms of the spectral density of horizontal ground vibrations $\ddot{y}_{0}(v)$. Rewriting (A 15) and including a damping term, $\mathrm{b}$, proportional to $\dot{\theta}$, we find

$\left\langle\dot{\theta}^{2}\right\rangle=\frac{\ddot{y}_{0}(v)^{2} d^{2}}{\left(d^{2}+R^{2} \sin ^{2} \psi\right)^{2}} \int_{0}^{\infty} \frac{\mathrm{d} \omega}{\left(\omega_{0}^{2}-\omega^{2}\right)^{2}+4 \gamma^{2} \omega^{2}}$,

where $\gamma=b / 4 m\left(d^{2}+R^{2} \sin ^{2} \psi\right)$.

On evaluation of the (standard) integral,

$$
\left\langle\theta^{2}\right\rangle=4 m^{2} d^{2} \ddot{y}_{0}(v)^{2} \cdot \frac{1}{4 b \cdot 2 m g d} .
$$

Finally we make the approximation that

$$
\left\langle\dot{\theta}^{2}\right\rangle \simeq \omega_{0}^{2}\left\langle\theta^{2}\right\rangle
$$

with $\omega_{0}^{2}=g d /\left(d^{2}+R^{2} \sin ^{2} \psi\right)$ and obtain from (A 18)

$$
\ddot{\psi}=\frac{\ddot{y}_{0}(v)^{2}}{8} \cdot \frac{Q}{g^{1 / 2}} \cdot \frac{d^{3 / 2}}{\left(d^{2}+R^{2} \sin ^{2} \psi\right)^{3 / 2}} \sin 2 \psi,
$$

where

$$
Q=\frac{2 m\left(d^{2}+R^{2} \sin ^{2} \psi\right) \omega_{0}}{b} .
$$


We shall assume that the simple pendulum mode is critically damped and calculate the maximum vibration spectral density permissible if we are to make a measurement of $G$ to a relative precision of $10^{-6}$.

Assuming the static deflection method $(\psi=\pi / 4)$, the parameters of the model experimental setup, and that the length of the fibre, $d$, is $0.5 \mathrm{~m}$ and the length of the beam, $2 R$, is $20 \mathrm{~cm}$, we can write

$$
2 m\left(d^{2}+R^{2} / 2\right) \ddot{\psi} \simeq \frac{2 G M m R}{r^{2}} \times 10^{-6} .
$$

Then using (A 21) we find

$$
\ddot{y}_{0}(v) \leqq 3 \times 10^{-7} \mathrm{~m} \mathrm{~s}^{-2} / \mathrm{Hz}^{1 / 2} \text {. }
$$

[1] S. L. Adler, Phys. Lett. B 95, 241 (1980).

[2] M. D. Pollock, Phys. Lett. B 132, 61 (1983).

[3] C. Mathiazhagan and V. B. John, Class. Quant. Grav. 1, L 29 (1984).

[4] V. A. Krat and I. L. Gerlovin, Sov. Phys. Dokl. 19, 107 (1974)

[5] E. J. Sternglass, Lett. Nuovo Cim. 41, 203 (1984).

[6] G. T. Gillies, Metrologia (in Press).

[7] BIPM Comptes Rendus $17^{\mathrm{e}}$ Conf. Gen. Poids et Mesures, 1983.

[8] A. de Rujula, CERN Preprint TH, 4466 (1986).

[9] J. Ellis, Nature, London 323,595 (1986).

[10] Y. Fujii, Nature Phys. Sci. 234, 5 (1971).

[11] Y. Fujii, Ann. Phys., New York 69, 494 (1972).

[12] S. C. Holding, F. D. Stacey, and G. J. Tuck, Phys. Rev. D 33, 3487 (1986).

[13] E. Fischbach, D. Sudarsky, A. Szafer, C. Talmadge, and S. H. Aronson, Phys. Rev. Letts. 56, 1, 3 (1986).

[14] G. Feinberg and J. Sucher, Phys. Rev. D 20, 1717 (1979).

[15] G. W. Gibbons and B. F. Whiting, Nature, London 291, 636 (1981).

[16] P. A. M. Dirac, Nature, London 139, 323 (1937).

[17] R. W. Hellings, P. J. Adams, J. D. Anderson, M. S. Keesey, E. L. Lau, E. M. Standish, V. M. Canuto, and I. Goldman, Phys. Rev. Lett. 51, 1609 (1983).

[18] R. C. Ritter and G. T. Gillies, Bull. Amer. Phys. Soc. 31, 805 (1986).

[19] R. C. Ritter, in: Proceedings of the Second Marcel Grossmann Meeting on General Relativity (ed. Ruffini, R.) 1039, North Holland, Amsterdam 1982.

[20] Y.-S. Wu and Z. Wang, Phys. Rev. Letts. 57, 16, 1978 (1986).

[21] E. R. Cohen and B. N. Taylor, CODATA Bulletin 63, 12 (1986).

[22] M. U. Sagitov, V. K. Milyukov, Y. A. Monakhov, V. S. Nazarenko, and K. G. Tadzhidinov, Dokl. Akad. Nauk. SSSR 245, 567 (1979).

[23] G. G. Luther and W. R. Towler, Phys. Rev. Lett. 48, 121 (1982).

[24] P. R. Heyl and P. Chrzanowski, Nat. Bur. Stand. (US) J. Res. 29, 1 (1942).

[25] C. Pontikis, C. R. Acad. Sci., Paris 274, 437 (1972).

[26] J. Zahradnicek, Phys. Z. 34, 126 (1933).

[27] O. V. Karagioz, V. V. Voronkov, V. P. Izmaylov, and N. I. Agafanov, Izv, Physics of the Solid Earth 11, $69(1975)$
For the time of swing method we can take the gravitational restoring torque signal as

$$
C_{\mathrm{G}}=\frac{2 G M m R(R+r)}{r^{3}},
$$

and with $\psi \ll 1$ (A 18) becomes

$$
\ddot{\psi}=\psi\left\langle\dot{\theta}^{2}\right\rangle \text {. }
$$

We can then write

$$
2 m d^{2} \frac{\ddot{\psi}}{\psi}=\frac{G M m R(R+r)}{r^{3}} \times 10^{-6}
$$

and find approximately the same result for the limit on $\ddot{y}_{0}(v)$.

[28] H. Bradner and M. Reichle, Am. Inst. of Aeronautics and Astronautics, Stanford Ca. (AIAA) Paper No. 72-819. AIAA Guidance and Control Conference (available from Technical Information Service 750 3rd Avenue, New York, NY 10017) (1972).

[29] P. G. Roll, R. Krotkov, and R. H. Dicke, Ann. Phys. New York 26, 442 (1964).

[30] V. B. Braginski and V. I. Panov, Sov. Phys. JETP 34, 463 (1972).

[31] R. Augustin, H. de Boer, H. Haars, and W. Michaelis, Feinwerktech. Messtech. 89, 280 (1981).

[32] J. W. Beams, A. R. Kuhlthau, R. A. Lowry, and H. M. Parker, Bull. Amer. Phys. Soc. 11, 249 (1965).

[33] W. A. Koldewyn and J. E. Faller, Bull. Amer. Phys. Soc. 17, 472 (1972)

[34] G. M. Keiser and J. E. Faller, Bull. Amer. Phys. Soc. 24, 579 (1979)

[35] J. Wilsing, Publ. d. Astrophysik. Observ. z. Potsdam 6, $35-127(1887)$.

[36] J. H. Poynting, Phil. Trans. Roy. Soc., London A 182, $565-656$ (1892).

[37] C. C. Speake and G. T. Gillies, Proc. Roy. Soc., London A (submitted for publication).

[38] N. A. Robertson, R. W. P. Drever, I. Kerr, and J. Hough, J. Phys. E: Sci. Instrum. 15, 1101 (1982).

[39] R. A. van Patten, in: Precision Measurement and Fundamental Constants II (ed. B. N. Taylor and W. D. Phillips), 659 (Nat. Bur. Stand. (US) Spec. Pub. 617, U.S. Gov. Printing Office, Washington, D.C., 1984).

[40] A. J. F. Metherell and T. J. Quinn, Metrologia 22, 87 (1986); 23, 119 (1986/87).

[41] D. Berman and R. L. Forward, in: Exploitation of Space for Experimental Research, 95, American Astronautical Society, Tarzana, California, 1968.

[42] P. Farinella, A. Milani, and A. M. Nobili, Astrophys. Space Sci. 73, 417 (1980).

[43] R. C. Ritter and G. T. Gillies, Bull. Amer. Phys. Soc. 29, 703 (1984).

[44] Y. Avron and M. Livio, Astrophys. J. 304, L 61 (1986).

[45] B. W. Petley, The Fundamental Physical Constants and the Frontier of Measurement, Adam Hilger, Bristol 1985. 